\title{
Medical Image of the Week: Pericardial Effusion in a Setting of Bacterial Endocarditis
}

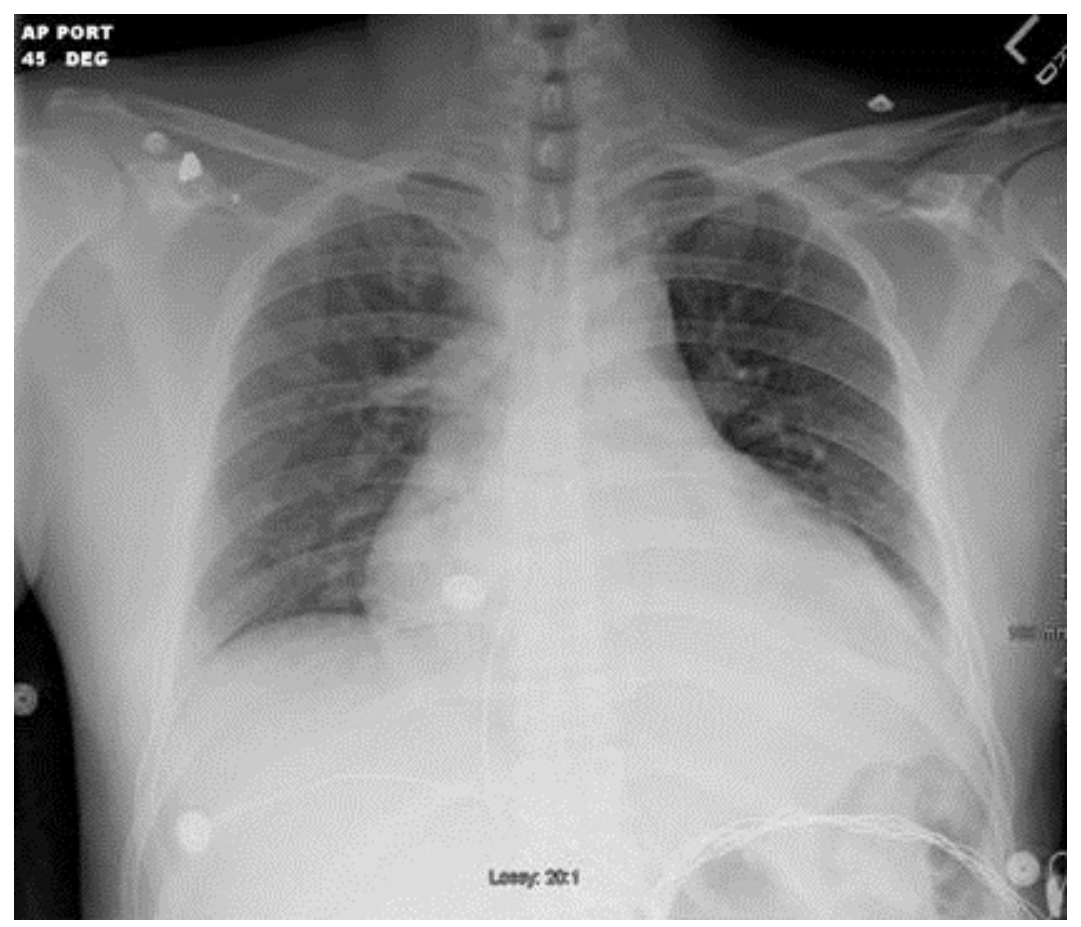

Figure 1. Single portable semi-upright chest radiograph with findings of an enlarged cardiomediastinal silhouette, and indistinctness of the perihilar vasculature.

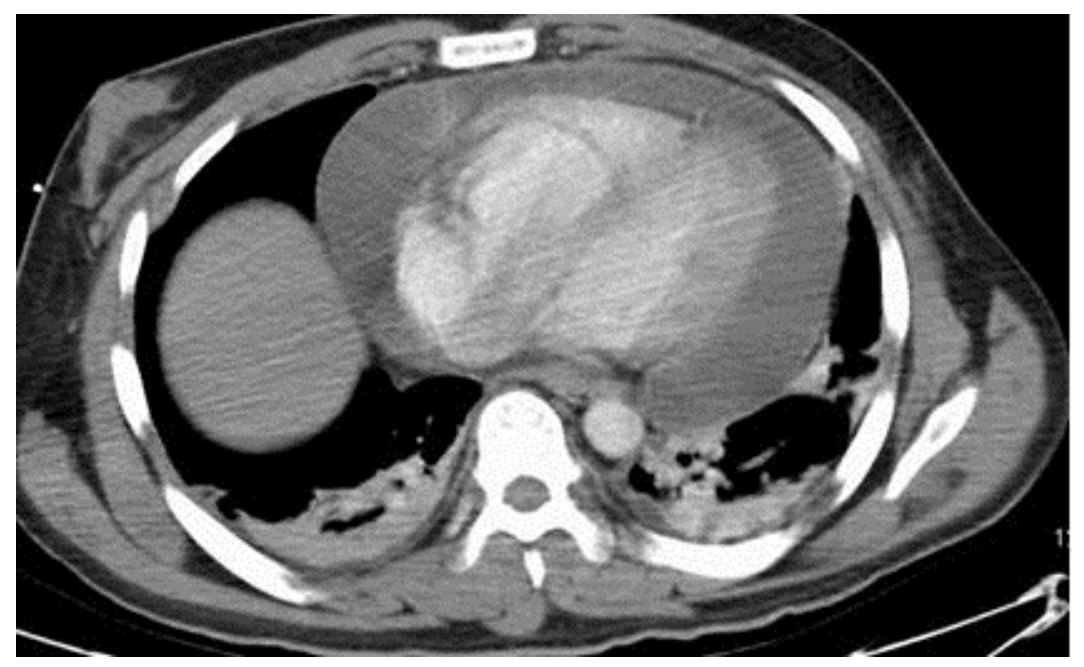

Figure 2. Axial contrast enhanced computed tomography-soft tissue windows. A large concentric rim (fluid density) surrounds all four chambers of the heart, consistent with a pericardial effusion. Notice how the right ventricle is normal, which can be collapsed in cardiac tamponade. 
A 25-year-old man with an extensive history of intravenous drug abuse presents to the hospital with worsening shortness of breath and fevers for two weeks. In the emergency department, he was initially provided breathing treatments including ipratropium/albuterol and methylprednisolone. As the patient still required supplemental oxygen, a chest radiograph was performed to evaluate for an underlying infectious etiology.

However, the chest radiograph portrayed an enlarged cardiomediastinal silhouette in a "water-bottle" appearance and obscuration of the hilar vessels (Figure 1). Given these findings, there was a high concern for a pericardial effusion, and the physicians opted for further cross-sectional imaging. The contrast enhanced computed tomography (CT) images confirmed the aforementioned diagnosis (Figure 2). As blood cultures eventually grew Staphylococcus aureus, and given the patient's extensive history of intravenous drug abuse, there was a high suspicion for bacterial endocarditis. A subsequent echocardiogram verified several valvular vegetations in keeping with endocarditis. The patient's vitals remained stable throughout the hospital course, and he was continued on long-term antibiotic therapy.

Chest radiographs are often unreliable in depicting pericardial effusions, as they require at least $200 \mathrm{~mL}$ of pericardial fluid to portray an enlarged cardiomediastinal silhouette (1). As fluid continues to accumulate in the pericardial space, the increase in pericardial pressure on the chambers can eventually lead to cardiac tamponade-a form of cardiogenic shock (2). Cardiac tamponade will result in a decrease in stroke volume, decreased blood pressure, and ultimately a diminished cardiac output; all of which require immediate intervention (2). Echocardiography remains the imaging modality of choice given its portability and high sensitivity in diagnosing pericardial fluid (3).

Amrit Hansra, MD

Department of Medical Imaging

University of Arizona

Tucson, AZ

\section{References}

1. Restrepo CS, Lemos DF, Lemos JA, et al. Imaging findings in cardiac tamponade with emphasis on CT. Radiographics. 2007 Nov-Dec;27(6):1595610. [CrossRef] [PubMed]

2. Spodick DH. Acute cardiac tamponade. N Engl J Med. 2003 Aug 14;349(7):684-90. [CrossRef] [PubMed]

3. Chong HH, Plotnick GD. Pericardial effusion and tamponade: evaluation, imaging modalities, and management. Compr Ther. 1995 Jul;21(7):378-85. [PubMed] 